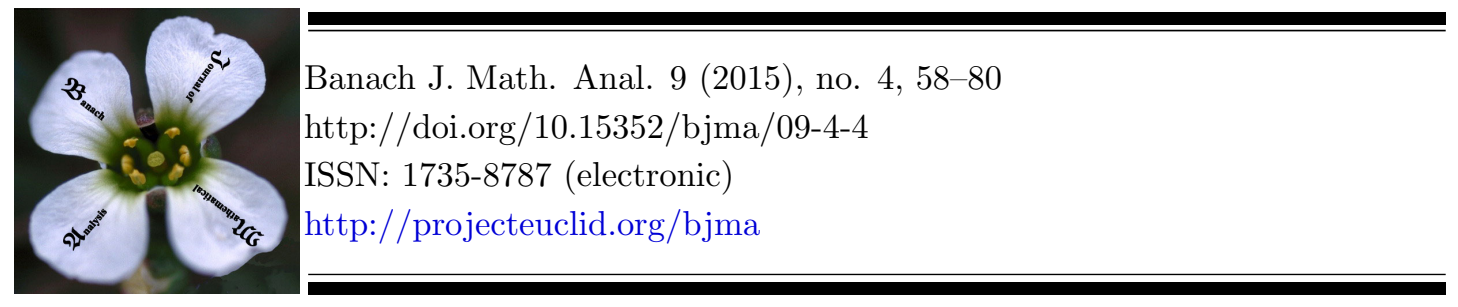

\title{
MULTIPLE GENERALIZED ANALYTIC FOURIER-FEYNMAN TRANSFORM VIA ROTATION OF GAUSSIAN PATHS ON FUNCTION SPACE
}

\author{
SEUNG JUN CHANG, JAE GIL CHOI* AND AE YOUNG KO \\ Communicated by P. E. Jorgensen
}

\begin{abstract}
The main purpose of this article is to develop the generalized analytic Fourier-Feynman transform theory. We introduce a generalized analytic Fourier-Feynman transform and a multiple generalized analytic FourierFeynman transform with respect to Gaussian processes on the function space $C_{a, b}[0, T]$ induced by a generalized Brownian motion process. We then establish a relationship between these two generalized analytic transforms.
\end{abstract}

\section{INTRODUCTION}

The main purpose of this article is to develop the generalized Fourier-Feynman transform theory, which is defined in terms of generalized Brownian motion processes. To explain what this transform is in its original context, let $H$ be the class of absolutely continuous paths $x$ from $[0, T]$ to $\mathbb{R}$ which start at 0 and with $D x \equiv d x / d t \in L^{2}[0, T]$, and let $\mathcal{D}$ be the non-existent Lebesgue measure on $H$. With in this heuristic context, the Fourier-Feynman transform of functionals $F$ on $H$ is defined by

$$
T_{-i q}(F)(y)=\exp \left\{\frac{i q}{2}\|y\|_{H}^{2}\right\} \int_{H} \exp \left\{-i q\langle x, y\rangle_{H}\right\} F(x) \frac{1}{Z_{q}} \exp \left\{\frac{i q}{2}\|x\|_{H}^{2}\right\} \mathcal{D}(d x)
$$

Date: Received: Sep. 22, 2014; Accepted: Dec. 12, 2014.

* Corresponding author.

2010 Mathematics Subject Classification. Primary 46G12; Secondary 28C20, 60J65, 60G15.

Key words and phrases. Generalized Brownian motion process, Gaussian process, generalized analytic Feynman integral, generalized analytic Fourier-Feynman transform, multiple generalized analytic Fourier-Feynman transform. 
for $q \in \mathbb{R} \backslash\{0\}$, where $Z_{q}$ is a certain ill defined normalization constant which is supposed to normalize $\frac{1}{Z_{q}} \exp \left\{\frac{i q}{2}\|x\|_{H}^{2}\right\} \mathcal{D}$ to be a probability measure.

Through the formal observation above, one can see that 'Fourier' refers to the $\exp \left\{-i q\langle x, y\rangle_{H}\right\}$ term while 'Feynman' refers to the $\exp \left\{\frac{i q}{2}\|x\|_{H}^{2}\right\}$ term in the integrand. Of course this is a very heuristic description. To head towards a rigorous definition, let $(H, W, \nu)$ be the abstract Wiener space with $H \hookrightarrow W$. For each $\lambda>0$, let us use the usual informal expression for Wiener measure with variance $\lambda^{-1}$ given by

$$
d \nu_{\lambda}(x)=\frac{1}{Z_{\lambda}} \exp \left\{-\frac{\lambda}{2}\|x\|_{H}^{2}\right\} \mathcal{D}(d x) .
$$

Then an informal evaluation shows that for $y \in H$,

$$
\begin{aligned}
& \exp \left\{-\frac{\lambda}{2}\|y\|_{H}^{2}\right\} \int_{H} F(x) \exp \left\{\lambda\langle x, y\rangle_{H}\right\} d \nu_{\lambda}(x) \\
& =\exp \left\{-\frac{\lambda}{2}\|y\|_{H}^{2}\right\} \int_{H} F(x) \exp \left\{\lambda\langle x, y\rangle_{H}\right\} \frac{1}{Z_{\lambda}} \exp \left\{-\frac{\lambda}{2}\|x\|_{H}^{2}\right\} \mathcal{D}(d x) \\
& =\int_{H} F(x) \frac{1}{Z_{\lambda}} \exp \left\{-\frac{1}{2}\|\sqrt{\lambda}(x-y)\|_{H}^{2}\right\} \mathcal{D}(d x) \\
& =\int_{H} F(x+y) \frac{1}{Z_{\lambda}} \exp \left\{-\frac{1}{2}\|\sqrt{\lambda} x\|_{H}^{2}\right\} \mathcal{D}(d x) \\
& =\int_{H} F\left(\lambda^{-1 / 2} x+y\right) \frac{1}{Z_{1}} \exp \left\{-\frac{1}{2}\|x\|_{H}^{2}\right\} \mathcal{D}(d x) \\
& =\int_{H} F\left(\lambda^{-1 / 2} x+y\right) d \nu(x) .
\end{aligned}
$$

Thus we should expect that for $y \in W$,

$$
T_{-i q}(F)(y)=\lim _{\lambda \rightarrow-i q} \int_{W} F\left(\lambda^{-1 / 2} x+y\right) d \nu(x),
$$

where one must first assume that $\lambda \rightarrow \int_{W} F\left(\lambda^{-1 / 2} x+y\right) d \nu(x)$ has an 'analytic continuation' in the right-half complex plane and that the above limit exists appropriately. Equation (1.1) with $y=0$ can be considered as the Feynman path integral which has been developed by many authors over many years.

Let $\left(C_{0}[0, T], \mathcal{M}, m_{w}\right)$ denote the classical Wiener space, where $C_{0}[0, T]$ is the space of real-valued continuous functions $x(t)$ on $[0, T]$ with $x(0)=0, \mathcal{M}$ is the class of all Wiener measurable subsets of $C_{0}[0, T]$, and $m_{w}$ is the Gaussian measure on $C_{0}[0, T]$ with mean zero and covariance function $r(s, t)=\min \{s, t\}$.

The concept of the 'analytic' Feynman integral on the Wiener space $C_{0}[0, T]$ was introduced by Cameron [2]. There is a great deal of published research on the analytic Feynman integral theory, and the analytic Fourier-Feynman transform on $C_{0}[0, T]$ has also been developed in the literature. In particular, the concept of the $L_{1}$ analytic Fourier-Feynman transform defined by the formula (1.1) on Wiener space $C_{0}[0, T]$ was introduced by Brue in [1]. Since then, the $L_{p}$ analytic Fourier-Feynman transform for $1 \leq p \leq 2$ was further developed by many mathematicians. For instance, see $[3,19]$. For an elementary introduction to the 
analytic Feynman integral and the analytic Fourier-Feynman transform, see [25] and the references cited therein.

On the other hand, the concepts of the analytic $\mathcal{Z}_{h^{-}}$Wiener integral (i.e., the Wiener integral with respect to Gaussian process $\mathcal{Z}_{h}$ ) and the analytic $\mathcal{Z}_{h^{-}}$ Feynman integral (i.e., the analytic Feynman integral with respect to Gaussian process $\left.\mathcal{Z}_{h}\right)$ on $C_{0}[0, T]$ were introduced by Chung, Park and Skoug in [12], and were further developed in $[5,10,22,23]$. In $[5,10,12,22,23]$, the $\mathcal{Z}_{h}$-Wiener integral was defined by the Wiener integral

$$
\int_{C_{0}[0, T]} F\left(\mathcal{Z}_{h}(x, \cdot)\right) d m_{w}(x)
$$

where $\mathcal{Z}_{h}(x, \cdot)$ is the Gaussian path given by the stochastic integral $\mathcal{Z}_{h}(x, t)=$ $\int_{0}^{t} h(s) d x(s)$ with $h \in L^{2}[0, T]$.

Next, let $D=[0, T]$ and let $(\Omega, \mathcal{B}, P)$ be a probability space. A generalized Brownian motion process (GBMP) on $\Omega \times D$ is an Gaussian process $Y \equiv\left\{Y_{t}\right\}_{t \in D}$ such that $Y_{0}=c$ almost everywhere for some constant $c \in \mathbb{R}$ (in this paper we set $c=0$ ), and for any $0 \leq s<t \leq T$,

$$
Y_{t}-Y_{s} \sim N(a(t)-a(s), b(t)-b(s)),
$$

where $N\left(m, \sigma^{2}\right)$ denotes the normal distribution with mean $m$ and variance $\sigma^{2}$, $a(t)$ is a continuous real-valued function on $[0, T]$, and $b(t)$ is a monotonically increasing continuous real-valued function on $[0, T]$. Thus, the GBMP $Y$ is determined by the functions $a(t)$ and $b(t)$. For more details, see [26, 27]. Note that when $a(t) \equiv 0$ and $b(t)=t$, the GBMP is a standard Brownian motion (Wiener process). We are obliged to point out that a standard Brownian motion is stationary in time, whereas a GBMP is generally not stationary in time, and is subject to a drift $a(t)$.

In $[6,8]$, the authors defined the generalized analytic Feynman integral and the generalized analytic Fourier-Feynman transform (GFFT) on the function space $C_{a, b}[0, T]$, and studied their properties and related topics. The function space $C_{a, b}[0, T]$, induced by a GBMP, was introduced by Yeh in [26], and was used extensively in $[6,7,8,9,13]$. There have also been several recent attempts to construct financial mathematical theories using this process $[11,14,16,21]$.

In this paper, we define a generalized analytic Feynman integral, a generalized analytic Fourier-Feynman transform, and a multiple generalized analytic FourierFeynman transform via a Gaussian process

$$
\mathcal{Z}_{h}(x, t)=\int_{0}^{t} h(s) d x(s), h \in L_{a, b}^{2}[0, T], x \in C_{a, b}[0, T]
$$

where $L_{a, b}^{2}[0, T]$ denote the separable Hilbert space associated with the continuous functions $a(\cdot)$ and $b(\cdot)$ that characterize the GBMP. We then investigate a rotation formula involving the two generalized analytic transforms on the general function space $C_{a, b}[0, T]$.

The Wiener process used in $[1,2,3,4,19]$ is stationary in time and is free of drift, while the Gaussian process used in $[5,10,12,22,23]$ is non-stationary in time and is free of drift. However, the stochastic process used in this paper, as 
well as in $[6,7,8,9,11,13,21,26]$, is non-stationary in time and is subject to a drift $a(t)$.

\section{Preliminaries}

In this section, we briefly list some of the preliminaries from $[6,7,8,9]$ that we need to establish our results in the next sections.

Let $a(t)$ be an absolutely continuous real-valued function on $[0, T]$ with $a(0)=0$ and $a^{\prime}(t) \in L^{2}[0, T]$, and let $b(t)$ be a strictly increasing, continuously differentiable real-valued function with $b(0)=0$ and $b^{\prime}(t)>0$ for each $t \in[0, T]$. The GBMP $Y$ determined by $a(t)$ and $b(t)$ is a Gaussian process with mean function $a(t)$ and covariance function $r(s, t)=\min \{b(s), b(t)\}$. For more details, see [6, 8]. By [27, Theorem 14.2], the probability measure $\mu$ induced by $Y$, taking a separable version, is supported by $C_{a, b}[0, T]$ (which is equivalent to the Banach space of continuous functions $x$ on $[0, T]$ with $x(0)=0$ under the sup norm). Hence, $\left(C_{a, b}[0, T], \mathcal{B}\left(C_{a, b}[0, T]\right), \mu\right)$ is the function space induced by $Y$ where $\mathcal{B}\left(C_{a, b}[0, T]\right)$ is the Borel $\sigma$-algebra of $C_{a, b}[0, T]$. We then complete this function space to obtain $\left(C_{a, b}[0, T], \mathcal{W}\left(C_{a, b}[0, T]\right), \mu\right)$ where $\mathcal{W}\left(C_{a, b}[0, T]\right)$ is the set of all $\mu$-Carathéodory measurable subsets of $C_{a, b}[0, T]$.

We note that the coordinate process defined by $e_{t}(x)=x(t)$ on $C_{a, b}[0, T] \times[0, T]$ is also the GBMP determined by $a(t)$ and $b(t)$. For more detailed studies about this function space $C_{a, b}[0, T]$, see $[6,8,26]$.

A subset $B$ of $C_{a, b}[0, T]$ is said to be scale-invariant measurable provided $\rho B$ is $\mathcal{W}\left(C_{a, b}[0, T]\right)$-measurable for all $\rho>0$, and a scale-invariant measurable set $N$ is said to be scale-invariant null provided $\mu(\rho N)=0$ for all $\rho>0$. A property that holds except on a scale-invariant null set is said to hold scale-invariant almost everywhere (s-a.e.). A functional $F$ is said to be scale-invariant measurable provided $F$ is defined on a scale-invariant measurable set and $F(\rho \cdot)$ is $\mathcal{W}\left(C_{a, b}[0, T]\right)$-measurable for every $\rho>0$. If two functionals $F$ and $G$ defined on $C_{a, b}[0, T]$ are equal s-a.e., we write $F \approx G$.

Let $L_{a, b}^{2}[0, T]$ be the space of functions on $[0, T]$ which are Lebesgue measurable and square integrable with respect to the Lebesgue-Stieltjes measures on $[0, T]$ induced by $a(\cdot)$ and $b(\cdot)$; i.e.,

$$
L_{a, b}^{2}[0, T]:=\left\{v: \int_{0}^{T} v^{2}(s) d b(s)<+\infty \text { and } \int_{0}^{T} v^{2}(s) d|a|(s)<+\infty\right\}
$$

where $|a|(\cdot)$ denotes the total variation function of the function $a(\cdot)$. Then $L_{a, b}^{2}[0, T]$ is a separable Hilbert space with inner product defined by

$$
(u, v)_{a, b}:=\int_{0}^{T} u(t) v(t) d m_{|a|, b}(t) \equiv \int_{0}^{T} u(t) v(t) d[b(t)+|a|(t)],
$$

where $m_{|a|, b}$ denotes the Lebesgue-Stieltjes measure induced by $|a|(\cdot)$ and $b(\cdot)$. In particular, note that $\|u\|_{a, b} \equiv \sqrt{(u, u)_{a, b}}=0$ if and only if $u(t)=0$ a.e. on $[0, T]$. Note that all functions of bounded variation on $[0, T]$ are elements of $L_{a, b}^{2}[0, T]$. 
The following Cameron-Martin subspace of $C_{a, b}[0, T]$ plays an important role throughout this paper. Let

$$
C_{a, b}^{\prime}[0, T]:=\left\{w \in C_{a, b}[0, T]: w(t)=\int_{0}^{t} z(s) d b(s) \text { for some } z \in L_{a, b}^{2}[0, T]\right\} .
$$

For $w \in C_{a, b}^{\prime}[0, T]$, with $w(t)=\int_{0}^{t} z(s) d b(s)$ for $t \in[0, T]$, let $D: C_{a, b}^{\prime}[0, T] \rightarrow$ $L_{a, b}^{2}[0, T]$ be defined by the formula

$$
D w(t):=z(t)=\frac{w^{\prime}(t)}{b^{\prime}(t)} .
$$

Then $C_{a, b}^{\prime} \equiv C_{a, b}^{\prime}[0, T]$ with inner product

$$
\left(w_{1}, w_{2}\right)_{C_{a, b}^{\prime}}:=\int_{0}^{T} D w_{1}(t) D w_{2}(t) d b(t)=\int_{0}^{T} z_{1}(t) z_{2}(t) d b(t) \equiv\left(z_{1}, z_{2}\right)_{0, b}
$$

is a separable Hilbert space.

Note that the two separable Hilbert spaces $L_{a, b}^{2}[0, T]$ and $C_{a, b}^{\prime}[0, T]$ are homeomorphic under the linear operator given by equation (2.1). The inverse operator of $D$ is given by

$$
\left(D^{-1} z\right)(t)=\int_{0}^{t} z(s) d b(s), \quad t \in[0, T] .
$$

Recall that above, as well as in papers $[6,7,8]$, we require that $a:[0, T] \rightarrow \mathbb{R}$ is an absolutely continuous function with $a(0)=0$ and with $\int_{0}^{T}\left|a^{\prime}(t)\right|^{2} d t<\infty$. Our conditions on $b:[0, T] \rightarrow \mathbb{R}$ imply that $b^{\prime}$ is continuous and $\delta<b^{\prime}(t)<\Delta$, $t \in[0, T]$, for some positive real numbers $\delta$ and $\Delta$. Hence we have

$$
\int_{0}^{T}\left|a^{\prime}(t)\right|^{2} d b(t)=\int_{0}^{T}\left|a^{\prime}(t)\right|^{2} b^{\prime}(t) d t<\infty .
$$

In this paper, in addition to the conditions put on $a(t)$ above, we add the condition

$$
\int_{0}^{T}\left|a^{\prime}(t)\right|^{2} d|a|(t)<\infty
$$

Then, the function $a:[0, T] \rightarrow \mathbb{R}$ satisfies the condition (2.2) if and only if $a$ is an element of $C_{a, b}^{\prime}[0, T]$. For more a detailed illustration for the condition (2.2), see [9].

For each $w \in C_{a, b}^{\prime}[0, T]$, the Paley-Wiener-Zygmund (PWZ) stochastic integral $(w, x)^{\sim}$ is given by the formula

$$
\begin{aligned}
(w, x)^{\sim}: & =\lim _{n \rightarrow \infty} \int_{0}^{T} \sum_{j=1}^{n}\left(w, g_{j}\right)_{C_{a, b}^{\prime}} D g_{j}(t) d x(t) \\
& =\lim _{n \rightarrow \infty} \int_{0}^{T} \sum_{j=1}^{n}\left(z, \alpha_{j}\right)_{0, b} \alpha_{j}(t) d x(t)
\end{aligned}
$$

for $\mu$-a.e. $x \in C_{a, b}[0, T]$ where $\left\{g_{j}\right\}_{j=1}^{\infty}$ is a complete orthonormal set in $C_{a, b}^{\prime}[0, T]$ such that for each $j \in \mathbb{N}, D g_{j}=\alpha_{j}$ is of bounded variation on $[0, T]$. 
Our definition of the PWZ stochastic integral is different than the definition given in $[6,7,8,13]$. But we will emphasis that the following fundamental facts are still true:

(i) It follows from the definition of the PWZ stochastic integral and from Parseval's equality that if $w \in C_{a, b}^{\prime}[0, T]$ and $x \in C_{a, b}^{\prime}[0, T]$, then $(w, x)^{\sim}$ exists and we have $(w, x)^{\sim}=(w, x)_{C_{a, b}^{\prime}}$.

(ii) If $D w=z \in L_{a, b}^{2}[0, T]$ is of bounded variation on $[0, T]$, then the PWZ stochastic integral $(w, x)^{\sim}$ equals the Riemann-Stieltjes integral $\int_{0}^{T} z(t) d x(t)$ for $\mu$-a.e. $x \in C_{a, b}[0, T]$.

(iii) The PWZ stochastic integral has the expected linearity properties. That is, for any real number $c, w \in C_{a, b}^{\prime}[0, T]$, and $x \in C_{a, b}[0, T]$, we have

$$
(w, c x)^{\sim}=c(w, x)^{\sim}=(c w, x)^{\sim} .
$$

From this, it follows that for each $w \in C_{a, b}^{\prime}[0, T],(w, x)^{\sim}$ exists for s-a.e. $x \in C_{a, b}[0, T]$.

(iv) For each $w \in C_{a, b}^{\prime}[0, T],(w, x)^{\sim}$ is a Gaussian random variable with mean $(w, a)_{C_{a, b}^{\prime}}$ and variance $\|w\|_{C_{a, b}^{\prime}}^{2}$. For all $w_{1}, w_{2} \in C_{a, b}^{\prime}[0, T]$, we have

$$
\int_{C_{a, b}[0, T]}\left(w_{1}, x\right)^{\sim}\left(w_{2}, x\right)^{\sim} d \mu(x)=\left(w_{1}, w_{2}\right)_{C_{a, b}^{\prime}}+\left(w_{1}, a\right)_{C_{a, b}^{\prime}}\left(w_{2}, a\right)_{C_{a, b}^{\prime}} .
$$

Thus, if $\left\{w_{1}, \ldots, w_{n}\right\}$ is an orthogonal set in $C_{a, b}^{\prime}[0, T]$, then the Gaussian random variables $\left(w_{j}, x\right)^{\sim}$ 's are independent.

For more details, see [9].

Throughout this paper, let $\mathbb{C}, \mathbb{C}_{+}$and $\widetilde{\mathbb{C}}_{+}$denote the set of complex numbers, complex numbers with positive real part, and nonzero complex numbers with nonnegative real part, respectively. For each $\lambda \in \mathbb{C}, \lambda^{1 / 2}$ denotes the principal square root of $\lambda$; i.e., $\lambda^{1 / 2}$ is always chosen to have nonnegative real part, so that $\lambda^{-1 / 2}=\left(\lambda^{-1}\right)^{1 / 2}$ is in $\mathbb{C}_{+}$for all $\lambda \in \widetilde{\mathbb{C}}_{+}$. We then have the following: for $\lambda \in \mathbb{C}$ with $\lambda=\alpha+i \beta$,

$$
\lambda^{-1 / 2} \equiv\left(\lambda^{-1}\right)^{1 / 2}=\sqrt{\frac{\sqrt{\alpha^{2}+\beta^{2}}+\alpha}{2\left(\alpha^{2}+\beta^{2}\right)}}-i \operatorname{sign}(\beta) \sqrt{\frac{\sqrt{\alpha^{2}+\beta^{2}}-\alpha}{2\left(\alpha^{2}+\beta^{2}\right)}},
$$

where $\operatorname{sign}(\beta)=1$ if $\beta \geq 0$ and $\operatorname{sign}(\beta)=-1$ if $\beta<0$.

\section{Gaussian PROCESSES}

For each $t \in[0, T]$, let $\mathrm{I}_{[0, t]}$ denote the indicator function of the interval $[0, t]$ and for $k \in C_{a, b}^{\prime}[0, T]$ with $D k=h$ and with $\|k\|_{C_{a, b}^{\prime}}=\left[\int_{0}^{T} h^{2}(t) d b(t)\right]^{1 / 2}>0$, let $\mathcal{Z}_{k}(x, t)$ be the PWZ stochastic integral

$$
\mathcal{Z}_{k}(x, t):=\left(D^{-1}\left(h \mathrm{I}_{[0, t]}\right), x\right)^{\sim} .
$$

Let

$$
\gamma_{k}(t):=\int_{0}^{t} D k(u) d a(u)=\int_{0}^{t} h(u) d a(u)
$$


and let

$$
\beta_{k}(t):=\int_{0}^{t}(D k(u))^{2} d b(u)=\int_{0}^{t} h^{2}(u) d b(u)
$$

Then the stochastic process $\mathcal{Z}_{k}: C_{a, b}[0, T] \times[0, T] \rightarrow \mathbb{R}$ is Gaussian with mean function

$$
\int_{C_{a, b}[0, T]} \mathcal{Z}_{k}(x, t) d \mu(x)=\int_{0}^{t} h(u) d a(u)=\gamma_{k}(t)
$$

and covariance function

$$
\begin{aligned}
& \int_{C_{a, b}[0, T]}\left(\mathcal{Z}_{k}(x, s)-\gamma_{k}(s)\right)\left(\mathcal{Z}_{k}(x, t)-\gamma_{k}(t)\right) d \mu(x) \\
& =\int_{0}^{\min \{s, t\}} h^{2}(u) d b(u)=\beta_{k}(\min \{s, t\}) .
\end{aligned}
$$

In addition, by [27, Theorem 21.1], $\mathcal{Z}_{k}(\cdot, t)$ is stochastically continuous in $t$ on $[0, T]$. If $h=D k$ is of bounded variation on $[0, T]$, then, for all $x \in C_{a, b}[0, T]$, $\mathcal{Z}_{k}(x, t)$ is continuous in $t$. Of course if $k(t) \equiv b(t)$, then $\mathcal{Z}_{b}(x, t)=x(t)$. Furthermore, if $a(t) \equiv 0$ and $b(t)=t$ on $[0, T]$, then the function space $C_{a, b}[0, T]$ reduces to the classical Wiener space $C_{0}[0, T]$ and the Gaussian process $(3.1)$ with $k(t) \equiv t$ is an ordinary Wiener process.

Let $C_{a, b}^{*}[0, T]$ be the set of functions $k$ in $C_{a, b}^{\prime}[0, T]$ such that $D k$ is continuous except for a finite number of finite jump discontinuities and is of bounded variation on $[0, T]$. For any $w \in C_{a, b}^{\prime}[0, T]$ and $k \in C_{a, b}^{*}[0, T]$, let the operation $\odot$ between $C_{a, b}^{\prime}[0, T]$ and $C_{a, b}^{*}[0, T]$ be defined by

$$
w \odot k:=D^{-1}(D w D k), \text { i.e., } D(w \odot k)=D w D k,
$$

where $D w D k$ denotes the pointwise multiplication of the functions $D w$ and $D k$. Then we observe the following algebraic structures:

- $C_{a, b}^{\prime}[0, T] \times C_{a, b}^{*}[0, T] \ni(w, k) \mapsto w \odot k=k \odot w \in C_{a, b}^{\prime}[0, T]$.

- For every $w \in C_{a, b}^{\prime}[0, T]$ and every $k_{1}, k_{2} \in C_{a, b}^{*}[0, T]$,

$$
\left(w \odot k_{1}\right) \odot k_{2}=w \odot\left(k_{1} \odot k_{2}\right) \text { and } w \odot\left(k_{1}+k_{2}\right)=w \odot k_{1}+w \odot k_{2} \text {. }
$$

- For every $w_{1}, w_{2} \in C_{a, b}^{\prime}[0, T]$ and every $k \in C_{a, b}^{*}[0, T]$,

$$
\left(w_{1}+w_{2}\right) \odot k=w_{1} \odot k+w_{2} \odot k .
$$

- For every $w_{1}, w_{2} \in C_{a, b}^{\prime}[0, T]$ and every $k \in C_{a, b}^{*}[0, T]$,

$$
\left(w_{1}, w_{2} \odot k\right)_{C_{a, b}^{\prime}}=\left(w_{1} \odot k, w_{2}\right)_{C_{a, b}^{\prime}} .
$$


We also observe that for $w \in C_{a, b}^{\prime}[0, T]$ and $k \in C_{a, b}^{*}[0, T]$,

$$
\begin{aligned}
\|w \odot k\|_{C_{a, b}^{\prime}} & =(w \odot k, w \odot k)_{C_{a, b}^{\prime}}^{1 / 2} \\
& =\left[\int_{0}^{T}\{D w(t)\}^{2}\{D k(t)\}^{2} d b(t)\right]^{1 / 2} \\
& \leq\|D k\|_{\infty}\left[\int_{0}^{T}\{D w(t)\}^{2} d b(t)\right]^{1 / 2} \\
& =\|D k\|_{\infty}\|w\|_{C_{a, b}^{\prime}},
\end{aligned}
$$

where $\|\cdot\|_{\infty}$ denotes the essential supremum norm. Furthermore, $\left(C_{a, b}^{*}[0, T], \odot\right)$ is a commutative algebra with the identity $b$.

For $w \in C_{a, b}^{\prime}[0, T]$ and $k \in C_{a, b}^{*}[0, T]$, it follows that

$$
\begin{aligned}
\left(w, \mathcal{Z}_{k}(x, \cdot)\right)^{\sim} & =\int_{0}^{T} D w(t) d\left(\int_{0}^{t} D k(s) d x(s)\right) \\
& =\int_{0}^{T} D w(t) D k(t) d x(t) \\
& =(w \odot k, x)^{\sim}
\end{aligned}
$$

for s-a.e $x \in C_{a, b}[0, T]$. Thus, throughout the rest of this paper, we require $k$ to be in $C_{a, b}^{*}[0, T]$ for each process $\mathcal{Z}_{k}$. This will ensure that the Lebesgue-Stieltjes integrals

$$
\|w \odot k\|_{C_{a, b}^{\prime}}^{2}=\int_{0}^{T}(D w(t))^{2}(D k(t))^{2} d b(t)
$$

and

$$
(w \odot k, a)_{C_{a, b}^{\prime}}=\int_{0}^{T} D w(t) D k(t) D a(t) d b(t)=\int_{0}^{T} D w(t) D k(t) d a(t)
$$

will exist for all $w \in C_{a, b}^{\prime}[0, T]$ and $k \in C_{a, b}^{*}[0, T]$.

Using equation (3.6), the change of variable theorem, and [7, equation (2.15)], we obtain the following integration formula: for $w \in C_{a, b}^{\prime}[0, T], k \in C_{a, b}^{*}[0, T]$ and $\zeta \in \mathbb{C}$

$$
\int_{C_{a, b}[0, T]} \exp \left\{\zeta\left(w, \mathcal{Z}_{k}(x, \cdot)\right)^{\sim}\right\} d \mu(x)=\exp \left\{\frac{\zeta^{2}}{2}\|w \odot k\|_{C_{a, b}^{\prime}}^{2}+\zeta(w \odot k, a)_{C_{a, b}^{\prime}}\right\} .
$$

\section{Generalized analytic Fourier-Feynman transform WITH RESPECT TO GAUSSIAN PROCESS}

Let $\mathcal{G}$ be a stochastically continuous Gaussian process on $C_{a, b}[0, T] \times[0, T]$. We define the $\mathcal{G}$-function space integral (namely, the function space integral with respect to the Gaussian paths $\mathcal{G}(x, \cdot))$ for functionals $F$ on $C_{a, b}[0, T]$ by the formula

$$
I_{\mathcal{G}}[F] \equiv I_{\mathcal{G}, x}[F(\mathcal{G}(x, \cdot))]:=\int_{C_{a, b}[0, T]} F(\mathcal{G}(x, \cdot)) d \mu(x)
$$

whenever the integral exists. 
Definition 4.1. Let $q$ be a nonzero real number and let $\Gamma$ be a connected neighborhood of $-i q$ in $\widetilde{\mathbb{C}}_{+}$such that $(0,+\infty) \cap \Gamma$ is an open interval of positive real numbers. Let $\mathcal{G}$ be a stochastically continuous Gaussian process on $C_{a, b}[0, T] \times[0, T]$, and let $F$ be a $\mathbb{C}$-valued scale-invariant measurable functional on $C_{a, b}[0, T]$ such that

$$
J_{F}(\mathcal{G} ; \lambda):=I_{\mathcal{G}, x}\left[F\left(\lambda^{-1 / 2} \mathcal{G}(x, \cdot)\right)\right]
$$

exists and is finite for all $\lambda>0$. If there exists a function $J_{F}^{*}(\mathcal{G} ; \lambda)$ analytic on $\operatorname{Int}(\Gamma)$, the interior of $\Gamma$ in $\widetilde{\mathbb{C}}_{+}$, such that $J_{F}^{*}(\mathcal{G} ; \lambda)=J_{F}(\mathcal{G} ; \lambda)$ for all $\lambda \in$ $(0,+\infty) \cap \Gamma$, then $J_{F}^{*}(\mathcal{G} ; \lambda)$ is defined to be the analytic $\mathcal{G}$-function space integral (namely, the analytic function space integral with respect to the paths $\mathcal{G}(x, \cdot)$ ) of $F$ over $C_{a, b}[0, T]$ with parameter $\lambda$, and for $\lambda \in \operatorname{Int}(\Gamma)$ we write

$$
I_{\mathcal{G}}^{\mathrm{an}_{\lambda}}[F] \equiv I_{\mathcal{G}, x}^{\mathrm{an}_{\lambda}}[F(\mathcal{G}(x, \cdot))] \equiv \int_{C_{a, b}[0, T]}^{\mathrm{an}_{\lambda}} F(\mathcal{G}(x, \cdot)) d \mu(x):=J_{F}^{*}(\mathcal{G} ; \lambda) .
$$

Next let $F$ be a measurable functional whose analytic $\mathcal{G}$-function space integral $I_{\mathcal{G}}^{\operatorname{an}_{\lambda}}[F]$ exists for all $\lambda \in \operatorname{Int}(\Gamma)$. If the following limit exists, we call it the generalized analytic $\mathcal{G}$-Feynman integral (the generalized analytic Feynman integral with respect to the paths $\mathcal{G}(x, \cdot))$ of $F$ with parameter $q$ and we write

$$
I_{\mathcal{G}}^{\operatorname{anf}_{q}}[F] \equiv I_{\mathcal{G}, x}^{\operatorname{anf}_{q}}[F(\mathcal{G}(x, \cdot))]:=\lim _{\lambda \rightarrow-i q} I_{\mathcal{G}, x}^{\mathrm{an}_{\lambda}}[F(\mathcal{G}(x, \cdot))],
$$

where $\lambda$ approaches $-i q$ through values in $\operatorname{Int}(\Gamma)$.

Next we state the definition of the generalized analytic Fourier-Feynman transform with respect to Gaussian process on function space.

Definition 4.2. Let $F$ be a scale-invariant measurable functional on $C_{a, b}[0, T]$, and let $\mathcal{G}$ be a stochastically continuous Gaussian process on $C_{a, b}[0, T] \times[0, T]$. Let $q$ be a nonzero real number, and let $\Gamma$ be a connected neighborhood of $-i q$ in $\widetilde{\mathbb{C}}_{+}$such that for all $\lambda \in \operatorname{Int}(\Gamma)$ and $y \in C_{a, b}[0, T]$, the following analytic $\mathcal{G}$-function space integral

$$
T_{\lambda, \mathcal{G}}(F)(y):=I_{\mathcal{G}, x}^{\mathrm{an}_{\lambda}}[F(y+\mathcal{G}(x, \cdot))]
$$

exists. For $p \in(1,2]$, we define the $L_{p}$ analytic $\mathcal{G}$-GFFT (namely, the GFFT with respect to the paths $\mathcal{G}(x, \cdot)), T_{q, \mathcal{G}}^{(p)}(F)$ of $F$, by the formula,

$$
T_{q, \mathcal{G}}^{(p)}(F)(y):=\underset{\substack{\lambda \rightarrow-i q \\ \lambda \in \operatorname{Int}(\Gamma)}}{\lim .} T_{\lambda, \mathcal{G}}(F)(y)
$$

if it exists; i.e., for each $\rho>0$,

$$
\lim _{\substack{\lambda \rightarrow-i q \\ \lambda \in \operatorname{Int}(\Gamma)}} \int_{C_{a, b}[0, T]}\left|T_{\lambda, \mathcal{G}}(F)(\rho y)-T_{q, \mathcal{G}}^{(p)}(F)(\rho y)\right|^{p^{\prime}} d \mu(y)=0
$$

where $1 / p+1 / p^{\prime}=1$. We define the $L_{1}$ analytic $\mathcal{G}$-GFFT, $T_{q, \mathcal{G}}^{(1)}(F)$ of $F$, by the formula

$$
T_{q, \mathcal{G}}^{(1)}(F)(y):=\lim _{\substack{\lambda \rightarrow-i q \\ \lambda \in \operatorname{Int}(\Gamma)}} T_{\lambda, \mathcal{G}}(F)(y)=I_{\mathcal{G}, x}^{\operatorname{anf}_{q}}[F(y+\mathcal{G}(x, \cdot))]
$$


if it exists.

We note that for $1 \leq p \leq 2, T_{q, \mathcal{G}}^{(p)}(F)$ is defined only s-a.e.. We also note that if $T_{q, \mathcal{G}}^{(p)}(F)$ exists and if $F \approx G$, then $T_{q, \mathcal{G}}^{(p)}(G)$ exists and $T_{q, \mathcal{G}}^{(p)}(G) \approx T_{q, \mathcal{G}}^{(p)}(F)$. Moreover, from equations (4.1), (4.2) and (4.3), it follows that

$$
I_{\mathcal{G}}^{\operatorname{anf}_{q}}[F] \equiv I_{\mathcal{G}, x}^{\operatorname{anf}_{q}}[F(\mathcal{G}(x, \cdot))]=T_{q, \mathcal{G}}^{(1)}(F)(0)
$$

in the sense that if either side exists, then both sides exist and equality holds.

Remark 4.3. Note that if $k \equiv b$ on $[0, T]$, then the generalized analytic $\mathcal{Z}_{b^{-}}$

Feynman integral, $I_{\mathcal{Z}_{b}}^{\text {anf }_{q}}[F]$, and the $L_{p}$ analytic $\mathcal{Z}_{b}$-GFFT, $T_{q, \mathcal{Z}_{b}}^{(p)}(F)$ agree with the previous definitions of the generalized analytic Feynman integral and the analytic GFFT respectively [6, 8].

Remark 4.4. When $\mathcal{G}=\mathcal{Z}_{k}$, we will simply denote $I_{\mathcal{Z}_{k}}[F], I_{\mathcal{Z}_{k}}^{\operatorname{an}_{\lambda}}[F], I_{\mathcal{Z}_{k}}^{\operatorname{anf}_{q}}[F]$, $T_{\lambda, \mathcal{Z}_{k}}(F)$ and $T_{q, \mathcal{Z}_{k}}^{(p)}(F)$ by $I_{k}[F], I_{k}^{\mathrm{an}_{\lambda}}[F], I_{k}^{\mathrm{anf}_{q}}[F], T_{\lambda, k}(F)$ and $T_{q, k}^{(p)}(F)$ respectively.

\section{FRESNEL TYPE CLASS}

We next introduce a Banach algebra of functionals on $C_{a, b}[0, T]$. Suppose that $\mathcal{M}\left(C_{a, b}^{\prime}[0, T]\right)$ is the space of $\mathbb{C}$-valued, countably additive measures defined on $\mathcal{B}\left(C_{a, b}^{\prime}[0, T]\right)$, the Borel class of $C_{a, b}^{\prime}[0, T]$. Then the measure $f$ in $\mathcal{B}\left(C_{a, b}^{\prime}[0, T]\right)$ necessarily has finite total variation $\|f\|$, and $\mathcal{M}\left(C_{a, b}^{\prime}[0, T]\right)$ is a Banach algebra under the norm $\|\cdot\|$ and with convolution as multiplication [15, 24].

The Fresnel type class $\mathcal{F}\left(C_{a, b}[0, T]\right)$ of functionals on $C_{a, b}[0, T]$ is defined as the space of all stochastic Fourier transforms of elements of $\mathcal{M}\left(C_{a, b}^{\prime}[0, T]\right)$; that is, $F \in \mathcal{F}\left(C_{a, b}[0, T]\right)$ if and only if there exists a measure $f$ in $\mathcal{M}\left(C_{a, b}^{\prime}[0, T]\right)$ such that

$$
F(x)=\int_{C_{a, b}^{\prime}[0, T]} \exp \left\{i(w, x)^{\sim}\right\} d f(w)
$$

for s-a.e. $x \in C_{a, b}[0, T]$. More precisely, since we shall identify functionals which coincide s-a.e. on $C_{a, b}[0, T], \mathcal{F}\left(C_{a, b}[0, T]\right)$ can be regarded as the space of all s-equivalence classes of functionals of the form (5.1).

The Fresnel type class $\mathcal{F}\left(C_{a, b}[0, T]\right)$ is a Banach algebra with norm

$$
\|F\|:=\|f\|=\int_{C_{a, b}^{\prime}[0, T]} d|f|(w) .
$$

In fact, the correspondence $f \mapsto F$ is injective, carries convolution into pointwise multiplication and is a Banach algebra isomorphism where $f$ and $F$ are related by (5.1).

For a positive real number $q_{0}, k \in C_{a, b}^{*}[0, T]$, and $w \in C_{a, b}^{\prime}[0, T]$, let

$$
\phi\left(q_{0}, k ; w\right):=\exp \left\{\left(2 q_{0}\right)^{-1 / 2}\|D k\|_{\infty}\|w\|_{C_{a, b}^{\prime}}\|a\|_{C_{a, b}^{\prime}}\right\}
$$

and let

$$
\Gamma_{q_{0}}:=\left\{\lambda \in \widetilde{\mathbb{C}}_{+}:\left|\operatorname{Im}\left(\lambda^{-1 / 2}\right)\right|<\left(2 q_{0}\right)^{-1 / 2}\right\}
$$


Also, for $\lambda \in \widetilde{\mathbb{C}}_{+}, k \in C_{a, b}^{*}[0, T]$, and $w \in C_{a, b}^{\prime}[0, T]$, let

$$
\psi(\lambda, k ; w):=\exp \left\{-\frac{1}{2 \lambda}\|w \odot k\|_{C_{a, b}^{\prime}}^{2}+i \lambda^{-1 / 2}(w \odot k, a)_{C_{a, b}^{\prime}}\right\} .
$$

Then, using Cauchy-Schwartz's inequality and (3.5), we observe that for all $\lambda \in$ $\Gamma_{q_{0}}$,

$$
|\psi(\lambda, k ; w)| \leq \exp \left\{\left|\operatorname{Im}\left(\lambda^{-1 / 2}\right)\right|\|w \odot k\|_{C_{a, b}^{\prime}}\|a\|_{C_{a, b}^{\prime}}\right\}<\phi\left(q_{0}, k ; w\right) .
$$

We note that for all real $q$ with $|q|>q_{0}$,

$$
(-i q)^{-1 / 2}=\frac{1}{\sqrt{2|q|}}+i \frac{\operatorname{sign}(q)}{\sqrt{2|q|}}
$$

by equation (2.3). Also a close examination of equation (5.3) shows that $-i q$ is an elements of the region $\Gamma_{q_{0}}$. More precisely, $-i q$ is an interior point of the region $\Gamma_{q_{0}}$ in $\widetilde{\mathbb{C}}_{+}$. Furthermore, $\Gamma_{q_{0}}$ is a connected neighborhood of $-i q$ in $\widetilde{\mathbb{C}}_{+}$, and $(0,+\infty) \subset \Gamma_{q_{0}}$.

For a positive real number $q_{0}$ and an element $k \in C_{a, b}^{*}[0, T]$, we define a subclass $\mathcal{F}_{k}^{q_{0}}$ of $\mathcal{F}\left(C_{a, b}[0, T]\right)$ by $F \in \mathcal{F}_{k}^{q_{0}}$ if and only if

$$
\int_{C_{a, b}^{\prime}[0, T]} \phi\left(q_{0}, k ; w\right) d|f|(w)<+\infty,
$$

where $f$ and $F$ are related by $(5.1)$ and $\phi\left(q_{0}, k ; w\right)$ is given by (5.2) above.

Remark 5.1. Recall that letting $a(t) \equiv 0$ and $b(t)=t$ on $[0, T]$, the function space $C_{a, b}[0, T]$ reduces to the classical Wiener space $C_{0}[0, T]$. In this case the Fresnel type class $\mathcal{F}\left(C_{a, b}[0, T]\right)$ reduces to the Fresnel class $\mathcal{F}\left(C_{0}[0, T]\right)$. Also, we see that $(w, a)_{C_{a, b}^{\prime}}=0$ for all $w \in C_{a, b}^{\prime}[0, T]=C_{0}^{\prime}[0, T]$. Hence for all $\lambda \in \widetilde{\mathbb{C}}_{+}$and all $k \in C_{0}^{*}[0, T],|\psi(\lambda, k ; w)| \leq \phi\left(q_{0}, k ; w\right) \equiv 1$ and so for any positive real number $q_{0}, \mathcal{F}_{k}^{q_{0}}=\mathcal{F}\left(C_{0}[0, T]\right)$.

It is known that $\mathcal{F}\left(C_{0}[0, T]\right)$ forms a Banach algebra over the complex field and that $\mathcal{F}\left(C_{0}[0, T]\right)$ is isometrically isomorphic to the Banach algebra $\mathcal{S}\left(L^{2}[0, T]\right)$ introduced by Cameron and Storvick; see [4, 17, 20]. For a more detailed study of Banach algebras of functionals on classical and abstract Wiener spaces, see [18, pp.609-629].

Theorem 5.2. Let $k$ be a nonzero element of $C_{a, b}^{*}[0, T]$, let $q_{0}$ be a positive real number, and let $F \in \mathcal{F}_{k}^{q_{0}}$ be given by equation (5.1). Then, for all real $q$ with $|q|>q_{0}$, the $L_{1}$ analytic $\mathcal{Z}_{k}-G F F T, T_{q, k}^{(1)}(F)$ of $F$ exists and is given by the formula

$$
T_{q, k}^{(1)}(F)(y)=\int_{C_{a, b}^{\prime}[0, T]} \exp \left\{i(w, y)^{\sim}\right\} \psi(-i q, k ; w) d f(w)
$$

for s-a.e. $y \in C_{a, b}[0, T]$, where $\psi$ is given by equation (5.4). 
Proof. Let $k \in C_{a, b}^{*}[0, T] \backslash\{0\}$ be given. Using (5.1), (3.6), the Fubini theorem, (3.7), and (5.4), we have that for all $\lambda>0$,

$$
\begin{aligned}
J_{F(y+\cdot)}\left(\mathcal{Z}_{k} ; \lambda\right) \equiv & I_{k, x}\left[F\left(y+\lambda^{-1 / 2} \mathcal{Z}_{k}(x, \cdot)\right)\right] \\
= & \int_{C_{a, b}^{\prime}[0, T]} \exp \left\{i(w, y)^{\sim}-\frac{1}{2 \lambda}\|w \odot k\|_{C_{a, b}^{\prime}}^{2}\right. \\
& \left.+i \lambda^{-1 / 2}(w \odot k, a)_{C_{a, b}^{\prime}}\right\} d f(w) \\
= & \int_{C_{a, b}^{\prime}[0, T]} \exp \left\{i(w, y)^{\sim}\right\} \psi(\lambda, k ; w) d f(w) .
\end{aligned}
$$

Next let $\Gamma_{q_{0}}$ be given by (5.3) and let $\operatorname{Int}\left(\Gamma_{q_{0}}\right)$ indicate the interior of the region $\Gamma_{q_{0}}$ in $\widetilde{\mathbb{C}}_{+}$. Also let

$$
J_{F(y+\cdot)}^{*}\left(\mathcal{Z}_{k} ; \lambda\right):=\int_{C_{a, b}^{\prime}[0, T]} \exp \left\{i(w, y)^{\sim}\right\} \psi(\lambda, k ; w) d f(w)
$$

for $\lambda \in \Gamma_{q_{0}}$. Then, under the condition $(5.6), J_{F(y+\cdot)}^{*}\left(\mathcal{Z}_{k} ; \cdot\right)$ is well defined, as a function of $\lambda$, and $J_{F(y+\cdot)}^{*}\left(\mathcal{Z}_{k} ; \lambda\right)=J_{F(y+\cdot)}\left(\mathcal{Z}_{k} ; \lambda\right)$ for all $\lambda>0$. Thus it suffices to show that $J_{F(y+\cdot)}^{*}\left(\mathcal{Z}_{k} ; \lambda\right)$ is an analytic function of $\lambda$ on $\operatorname{Int}\left(\Gamma_{q_{0}}\right)$ to establish the desired result. We will use the Morera theorem to show that $J_{F(y+\cdot)}^{*}\left(\mathcal{Z}_{k} ; \lambda\right)$ is an analytic function of $\lambda$ on $\operatorname{Int}\left(\Gamma_{q_{0}}\right)$. For each $\lambda \in \operatorname{Int}\left(\Gamma_{q_{0}}\right)$, let $\left\{\lambda_{l}\right\}_{l=1}^{\infty}$ be a sequence in $\mathbb{C}_{+}$which converges to $\lambda$. Then, for every $l \in \mathbb{N}, \operatorname{Re}\left(\lambda_{l}^{-1 / 2}\right)>\left|\operatorname{Im}\left(\lambda_{l}^{-1 / 2}\right)\right| \geq 0$, and so

$$
\begin{aligned}
& \left|\exp \left\{i(w, y)^{\sim}\right\} \psi(\lambda, k ; w)\right|=|\psi(\lambda, k ; w)| \\
& =\left|\exp \left\{-\frac{1}{2 \lambda_{l}}\|w \odot k\|_{C_{a, b}^{\prime}}^{2}+i \lambda_{l}^{-1 / 2}(w \odot k, a)_{C_{a, b}^{\prime}}\right\}\right| \\
& =\mid \exp \left\{-\frac{1}{2}\left(\left[\operatorname{Re}\left(\lambda_{l}^{-1 / 2}\right)\right]^{2}-\left[\operatorname{Im}\left(\lambda_{l}^{-1 / 2}\right)\right]^{2}\right.\right. \\
& \left.+i 2 \operatorname{Re}\left(\lambda_{l}^{-1 / 2}\right) \operatorname{Im}\left(\lambda_{l}^{-1 / 2}\right)\right)\|w \odot k\|_{C_{a, b}^{\prime}}^{2} \\
& \left.\quad+i\left(\operatorname{Re}\left(\lambda_{l}^{-1 / 2}\right)+i \operatorname{Im}\left(\lambda_{l}^{-1 / 2}\right)\right)(w \odot k, a)_{C_{a, b}^{\prime}}\right\} \mid \\
& \leq \exp \left\{-\operatorname{Im}\left(\lambda_{l}^{-1 / 2}\right)(w \odot k, a)_{C_{a, b}^{\prime}}\right\} \\
& \leq \exp \left\{\left|\operatorname{Im}\left(\lambda_{l}^{-1 / 2}\right)\right|\|w \odot k\|_{C_{a, b}^{\prime}}\|a\|_{C_{a, b}^{\prime}}\right\} .
\end{aligned}
$$

Since $\lambda_{l}^{-1 / 2} \rightarrow \lambda^{-1 / 2}$ for given $\lambda \in \operatorname{Int}\left(\Gamma_{q_{0}}\right)$, there exists a sufficiently large $L \in \mathbb{N}$ such that $\left|\operatorname{Im}\left(\lambda_{l}^{-1 / 2}\right)\right|<1 / \sqrt{2 q_{0}}$ for every $l \geq L$, and so by the inequalities (5.5) 
and (5.6),

$$
\begin{aligned}
& \int_{C_{a, b}^{\prime}[0, T]}\left|\exp \left\{i(w, y)^{\sim}\right\} \psi\left(\lambda_{l}, k ; w\right)\right| d|f|(w) \\
& \leq \int_{C_{a, b}^{\prime}[0, T]} \exp \left\{\left|\operatorname{Im}\left(\lambda_{l}^{-1 / 2}\right)\right|\|w \odot k\|_{C_{a, b}^{\prime}}\|a\|_{C_{a, b}^{\prime}}\right\} d|f|(w) \\
& =\int_{C_{a, b}^{\prime}[0, T]} \phi\left(q_{0}, k ; w\right) d|f|(w)<+\infty
\end{aligned}
$$

whenever $l \geq L$. Thus, applying the dominated convergence theorem, we see that $J_{F(y+\cdot)}^{*}\left(\mathcal{Z}_{k} ; \lambda\right)$ is a continuous function of $\lambda$ on $\operatorname{Int}\left(\Gamma_{q_{0}}\right)$. Since $g(\lambda) \equiv$ $\exp \left\{i(w, y)^{\sim}\right\} \psi(\lambda, k ; w)$ is analytic on $\operatorname{Int}\left(\Gamma_{q_{0}}\right)$, applying the Fubini theorem, it follows that

$$
\int_{\triangle} J_{F(y+\cdot)}^{*}\left(\mathcal{Z}_{k} ; \lambda\right) d \lambda=\int_{C_{a, b}^{\prime}[0, T]} \int_{\triangle} g(\lambda) d \lambda d f(w)=0
$$

for all rectifiable simple closed curve $\triangle$ lying in $\operatorname{Int}\left(\Gamma_{q_{0}}\right)$. Thus by the Morera theorem, $J_{F(y+\cdot)}^{*}\left(\mathcal{Z}_{k} ; \lambda\right)$ is analytic on $\operatorname{Int}\left(\Gamma_{q_{0}}\right)$. Therefore, the analytic $\mathcal{Z}_{k}$-function space integral $T_{\lambda, k}(F)(y) \equiv I_{k, x}^{\mathrm{an}_{\lambda}}\left[F\left(y+\mathcal{Z}_{k}(x, \cdot)\right)\right]=J_{F(y+\cdot)}^{*}\left(\mathcal{Z}_{k} ; \lambda\right)$ exists and is given by the right hand side of (5.8).

Finally, using (4.3), (5.8), and the dominated convergence theorem (the use of which is justified by (5.9) for a sequence $\left\{\lambda_{l}\right\}$ with $\lambda \rightarrow-i q$ ), it follows that for s-a.e. $y \in C_{a, b}[0, T]$,

$$
\begin{aligned}
T_{q, k}^{(1)}(F)(y) & =\lim _{\substack{\lambda \rightarrow-i q \\
\lambda \in \operatorname{Int}(\Gamma)}} T_{\lambda, k}(F)(y) \\
& =\lim _{\substack{\lambda \rightarrow-i q \\
\lambda \in \operatorname{Int}(\Gamma)}} \int_{C_{a, b}^{\prime}[0, T]} \exp \left\{i(w, y)^{\sim}\right\} \psi(\lambda, k ; w) d f(w) \\
& =\int_{C_{a, b}^{\prime}[0, T]} \exp \left\{i(w, y)^{\sim}\right\} \lim _{\substack{\lambda \rightarrow-i q \\
\lambda \in \operatorname{Int}(\Gamma)}} \psi(\lambda, k ; w) d f(w) \\
& =\int_{C_{a, b}^{\prime}[0, T]} \exp \left\{i(w, y)^{\sim}\right\} \psi(-i q, k ; w) d f(w)
\end{aligned}
$$

as desired.

By a careful examination of the proof of Theorem 5.2 and equation (4.4), we obtain the following corollary.

Corollary 5.3. Let $k, q_{0}$ and $F$ be as in Theorem 5.2. Then,

(i) for each $\lambda$ in $\operatorname{Int}\left(\Gamma_{q_{0}}\right)$, the analytic $\mathcal{Z}_{k}$-function space integral of $F, I_{k}^{\mathrm{an}_{\lambda}}[F]$ exists and is given by the formula

$$
I_{k}^{\mathrm{an}_{\lambda}}[F]=\int_{C_{a, b}^{\prime}[0, T]} \psi(\lambda, k ; w) d f(w)
$$

; and 
(ii) for all real $q$ with $|q|>q_{0}$, the generalized analytic $\mathcal{Z}_{k}$-Feynman integral of $F, I_{k}^{\operatorname{anf}_{q}}[F]$ exists and is given by the formula

$$
I_{k}^{\operatorname{anf}_{q}}[F]=T_{q, k}^{(1)}(F)(0)=\int_{C_{a, b}^{\prime}[0, T]} \psi(-i q, k ; w) d f(w) .
$$

Theorem 5.4. Let $k, q_{0}$ and $F$ be as in Theorem 5.2. Then for all $p \in(1,2]$ and all real $q$ with $|q|>q_{0}$, the $L_{p}$ analytic $\mathcal{Z}_{k}-G F F T$ of $F, T_{q, k}^{(p)}(F)$ exists and is given by the right hand side of equation (5.7) for s-a.e. $y \in C_{a, b}[0, T]$.

Proof. Let $\Gamma_{q_{0}}$ be given by equation (5.3). It was shown in the proof of Theorem 5.2 that $T_{\lambda, k}(F)(y)$ is an analytic function of $\lambda$ throughout the $\operatorname{domain} \operatorname{Int}\left(\Gamma_{q_{0}}\right)$ in $\mathbb{C}_{+}$. In view of Definition 4.2 , it will suffice to show that for each $\rho>0$,

$$
\lim _{\substack{\lambda \rightarrow-i q \\ \lambda \in \operatorname{Int}(\Gamma)}} \int_{C_{a, b}[0, T]}\left|T_{\lambda, k}(F)(\rho y)-T_{q, k}^{(p)}(F)(\rho y)\right|^{p^{\prime}} d \mu(y)=0 .
$$

Fixing $p \in(1,2]$, and using the inequalities (5.5) and (5.6), we obtain that for all $\rho>0$ and all $\lambda \in \operatorname{Int}\left(\Gamma_{q_{0}}\right)$,

$$
\begin{aligned}
& \left|T_{\lambda, k}(F)(y)-T_{q, k}^{(p)}(F)(y)\right|^{p^{\prime}} \\
& \leq\left|\int_{C_{a, b}^{\prime}[0, T]} \exp \left\{i(w \odot k, y)^{\sim}\right\}[\psi(\lambda, k ; w)-\psi(-i q, k ; w)] d f(w)\right|^{p^{\prime}} \\
& \leq\left(\int_{C_{a, b}^{\prime}[0, T]}[|\psi(\lambda, k ; w)|+|\psi(-i q, k ; w)|] d|f|(w)\right)^{p^{\prime}} \\
& \leq\left(2 \int_{C_{a, b}^{\prime}[0, T]} \phi\left(q_{0}, k ; w\right) d|f|(w)\right)^{p^{\prime}}<+\infty .
\end{aligned}
$$

Hence by the dominated convergence theorem, it follows that for each $p \in(1,2]$ and each $\rho>0$,

$$
\begin{aligned}
& \lim _{\substack{\lambda \rightarrow-i q \\
\lambda \in \operatorname{Int}(\Gamma)}} \int_{C_{a, b}[0, T]}\left|T_{\lambda, k}(F)(\rho y)-T_{q, k}^{(p)}(F)(\rho y)\right|^{p^{\prime}} d \mu(y) \\
& =\lim _{\substack{\lambda \rightarrow-i q \\
\lambda \in \operatorname{Int}(\Gamma)}} \mid \int_{C_{a, b}^{\prime}[0, T]} \exp \left\{i(w \odot k, \rho y)^{\sim}\right\} \psi(\lambda, k ; w) d f(w) \\
& \quad-\left.\int_{C_{a, b}^{\prime}[0, T]} \exp \left\{i(w \odot k, \rho y)^{\sim}\right\} \psi(-i q, k ; w) d f(w)\right|^{p^{\prime}} d \mu(y) \\
& =\int_{C_{a, b}[0, T]} \mid \int_{C_{a, b}^{\prime}[0, T]} \exp \left\{i(w \odot k, \rho y)^{\sim}\right\} \\
& \quad \times\left.\lim _{\substack{\lambda \rightarrow-i q \\
\lambda \in \operatorname{Int}(\Gamma)}}[\psi(\lambda, k ; w)-\psi(-i q, k ; w)] d f(w)\right|^{p^{\prime}} d \mu(y) \\
& =0
\end{aligned}
$$


and the theorem is proved.

Remark 5.5. Let $k, q_{0}$ and $F$ be as in Theorem 5.2. For a real number $q$ with $|q|>q_{0}$, define a set function $f_{q, k}: \mathcal{B}\left(C_{a, b}^{\prime}[0, T]\right) \rightarrow \mathbb{C}$ by

$$
f_{q, k}(B):=\int_{B} \psi(-i q, k ; w) d f(w), \quad B \in \mathcal{B}\left(C_{a, b}^{\prime}[0, T]\right),
$$

where $f$ and $F$ are related by equation (5.1). Then it is obvious that $f_{q, k}$ belongs to $\mathcal{M}\left(C_{a, b}^{\prime}[0, T]\right)$, and for all $p \in(1,2], T_{q, k}^{(p)}(F)$ can be expressed as

$$
T_{q, k}^{(1)}(F)(y)=T_{q, k}^{(p)}(F)(y)=\int_{C_{a, b}^{\prime}[0, T]} \exp \left\{i(w, y)^{\sim}\right\} d f_{q, k}(w)
$$

for s-a.e. $y \in C_{a, b}[0, T]$. Hence $T_{q, k}^{(p)}(F)$ belongs to $\mathcal{F}\left(C_{a, b}^{\prime}[0, T]\right)$ for all $p \in[1,2]$.

\section{ITERATED GENERALIZED FOURIER-FEYNMAN TRANSFORM WITH GaUsSian PATHS}

For a positive real number $q_{0}$, a finite subset $\left\{k_{1}, \ldots, k_{n}\right\}$ of $C_{a, b}^{*}[0, T] \backslash\{0\}$, and $w \in C_{a, b}^{\prime}[0, T]$, let

$$
\begin{aligned}
\Phi\left(q_{0}, k_{1}, \ldots, k_{n} ; w\right): & =\prod_{j=1}^{n} \phi\left(q_{0}, k_{j} ; w\right) \\
& =\exp \left\{\frac{\|w\|_{C_{a, b}^{\prime}}\|a\|_{C_{a, b}^{\prime}}}{\sqrt{2 q_{0}}} \sum_{j=1}^{n}\left\|D k_{j}\right\|_{\infty}\right\},
\end{aligned}
$$

where $\phi$ is given by (5.2). Also, for $\lambda_{1}, \ldots, \lambda_{n} \in \widetilde{\mathbb{C}}_{+}, k_{1}, \ldots, k_{n} \in C_{a, b}^{*}[0, T] \backslash\{0\}$, and $w \in C_{a, b}^{\prime}[0, T]$, let

$$
\begin{aligned}
& \Psi_{n}\left(\lambda_{1} \ldots, \lambda_{n}, k_{1}, \ldots, k_{n} ; w\right):=\prod_{j=1}^{n} \psi\left(\lambda_{j}, k_{j} ; w\right) \\
& \quad=\exp \left\{-\sum_{j=1}^{n} \frac{1}{2 \lambda_{j}}\left\|w \odot k_{j}\right\|_{C_{a, b}^{\prime}}^{2}+\sum_{j=1}^{n} i \lambda_{j}^{-1 / 2}\left(w \odot k_{j}, a\right)_{C_{a, b}^{\prime}}\right\},
\end{aligned}
$$

where $\psi$ is given by (5.4). Then, using (3.5), we observe that for all $\lambda_{1}, \ldots, \lambda_{n} \in$ $\Gamma_{q_{0}}$

$$
\begin{aligned}
& \left|\Psi_{n}\left(\lambda_{1} \ldots, \lambda_{n}, k_{1}, \ldots, k_{n} ; w\right)\right| \\
& \leq \exp \left\{\sum_{j=1}^{n}\left|\operatorname{Im}\left(\lambda_{j}^{-1 / 2}\right)\right|\left\|w \odot k_{j}\right\|_{C_{a, b}^{\prime}}\|a\|_{C_{a, b}^{\prime}}\right\} \\
& <\Phi\left(q_{0}, k_{1}, \ldots, k_{n} ; w\right) .
\end{aligned}
$$

Given a positive real number $q_{0}$ and a finite subset $\left\{k_{1}, \ldots, k_{n}\right\}$ of $C_{a, b}^{*}[0, T] \backslash$ $\{0\}$, we define a subclass $\mathcal{F}_{k_{1}, \ldots, k_{n}}^{q_{0}}$ of $\mathcal{F}\left(C_{a, b}[0, T]\right)$ by $F \in \mathcal{F}_{k_{1}, \ldots, k_{n}}^{q_{0}}$ if and only if

$$
\int_{C_{a, b}^{\prime}[0, T]} \Phi\left(q_{0}, k_{1}, \ldots, k_{n} ; w\right) d|f|(w)<+\infty,
$$


where $f$ and $F$ are related by equation (5.1) and $\Phi$ is given by equation (6.1).

From (5.6), (5.2), (6.1), and (6.3), one can see that the class $\mathcal{F}_{k_{1}, \ldots, k_{n}}^{q_{0}}$ is a subclass of $\mathcal{F}_{k_{j}}^{q_{0}}$ for each $j \in\{1, \ldots, n\}$. Furthermore we have the chain

$$
\mathcal{F}_{k_{1}, \ldots, k_{n}}^{q_{0}} \equiv \mathcal{F}_{k_{\pi(1)}, \ldots, k_{\pi(n)}}^{q_{0}} \subset \mathcal{F}_{k_{\pi(1)}, \ldots, k_{\pi(n-1)}}^{q_{0}} \subset \mathcal{F}_{k_{\pi(1)}, \ldots, k_{\pi(n-2)}}^{q_{0}} \subset \cdots \subset \mathcal{F}_{k_{\pi(1)}}
$$

for any permutation $\pi$ of $\{1, \ldots, n\}$.

In view of Theorems 5.2, 5.4, and Remark 5.5, we obtain the following theorem.

Theorem 6.1. Let $\left\{k_{1}, \ldots, k_{n}\right\}$ be nonzero elements of $C_{a, b}^{*}[0, T]$, let $q_{0}$ be a positive real number, and let $F \in \mathcal{F}_{k_{1}, \ldots, k_{n}}^{q_{0}}$ be given by equation (5.1). Then, for every $p \in[1,2]$, all real numbers $q_{1}, \ldots, q_{n}$ with $\left|q_{j}\right|>q_{0}, j \in\{1, \ldots, n\}$, the iterated GFFT, $T_{q_{n}, k_{n}}^{(p)}\left(\cdots\left(T_{q_{1}, k_{1}}^{(p)}(F)\right) \cdots\right)$ of $F$ exists and is given by the formula

$$
\begin{aligned}
& T_{q_{n}, k_{n}}^{(p)}\left(\cdots\left(T_{q_{1}, k_{1}}^{(p)}(F)\right) \cdots\right)(y) \\
& =\int_{C_{a, b}^{\prime}[0, T]} \exp \left\{i(w, y)^{\sim}\right\} \Psi_{n}\left(-i q_{1}, \ldots,-i q_{2}, k_{1}, \ldots, k_{n} ; w\right) d f(w)
\end{aligned}
$$

for s-a.e. $y \in C_{a, b}[0, T]$, where $\Psi_{n}$ is given by equation (6.2).

\section{Multiple Generalized Fourier-Feynman transform With GAUSSIAN PATHS}

In this section, we define a multiple generalized analytic Fourier-Feynman

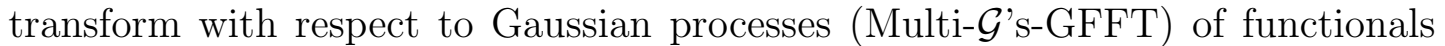
on $C_{a, b}[0, T]$. We then establish a relationship between the analytic Multi-G'sGFFT and the analytic $\mathcal{G}$-GFFT.

Let $F$ be a scale-invariant measurable functional on $C_{a, b}[0, T]$ and let $\mathcal{G}_{j}, j \in$ $\{1, \ldots, n\}$, be stochastically continuous Gaussian processes on $C_{a, b}[0, T] \times[0, T]$. For $\lambda>0$ and $y \in C_{a, b}[0, T]$, define a transform $\mathcal{M}_{\lambda,\left(\mathcal{G}_{1}, \ldots, \mathcal{G}_{n}\right)}(F)$ as follows:

$$
\begin{aligned}
& \mathcal{M}_{\lambda,\left(\mathcal{G}_{1}, \ldots, \mathcal{G}_{n}\right)}(F)(y) \\
:= & \int_{C_{a, b}^{n}[0, T]} F\left(y+\lambda^{-1 / 2} \sum_{j=1}^{n} \mathcal{G}_{j}\left(x_{j}, \cdot\right)\right) d \mu^{n}\left(x_{1}, \ldots, x_{n}\right) .
\end{aligned}
$$

Let $q$ be a nonzero real number, and let $\Gamma$ be a connected neighborhood of $-i q$ in $\widetilde{\mathbb{C}}_{+}$such that $(0,+\infty) \cap \Gamma$ is an open interval of positive real numbers. Let $\mathcal{M}_{\lambda,\left(\mathcal{G}_{1}, \ldots, \mathcal{G}_{n}\right)}(F)(y)$ again denote an analytic extension of $\mathcal{M}_{\lambda,\left(\mathcal{G}_{1}, \ldots, \mathcal{G}_{n}\right)}(F)(y)$ as a function of $\lambda$ in $\operatorname{Int}(\Gamma)$. For $p \in(1,2]$, we define the $L_{p}$ analytic Multi$\left(\mathcal{G}_{1}, \ldots, \mathcal{G}_{n}\right)$-GFFT, $\mathcal{M}_{q,\left(\mathcal{G}_{1}, \ldots, \mathcal{G}_{n}\right)}^{(p)}(F)$ of $F$, by the formula

$$
\mathcal{M}_{q,\left(\mathcal{G}_{1}, \ldots, \mathcal{G}_{n}\right)}^{(p)}(F)(y):=\lim _{\substack{\lambda \rightarrow-i q \\ \lambda \in \operatorname{Int}(\Gamma)}} \mathcal{M}_{\lambda,\left(\mathcal{G}_{1}, \ldots, \mathcal{G}_{n}\right)}(F)(y)
$$

if it exists. We also define the $L_{1}$ analytic Multi- $\left(\mathcal{G}_{1}, \ldots, \mathcal{G}_{n}\right)-\operatorname{GFFT}, \mathcal{M}_{q,\left(\mathcal{G}_{1}, \ldots, \mathcal{G}_{n}\right)}^{(1)}(F)$ of $F$, by the formula

$$
\mathcal{M}_{q,\left(\mathcal{G}_{1}, \ldots, \mathcal{G}_{n}\right)}^{(1)}(F)(y):=\lim _{\substack{\lambda \rightarrow-i q \\ \lambda \in \operatorname{Int}(\Gamma)}} \mathcal{M}_{\lambda,\left(\mathcal{G}_{1}, \ldots, \mathcal{G}_{n}\right)}(F)(y)
$$


if it exists.

Clearly, we have that

$$
\mathcal{M}_{\lambda,\left(\mathcal{G}_{1}\right)}(F)(y)=T_{\lambda, \mathcal{G}_{1}}(F)(y)
$$

for all $\lambda \in \operatorname{Int}(\Gamma)$, and

$$
\mathcal{M}_{q,\left(\mathcal{G}_{1}\right)}^{(p)}(F)(y)=T_{q, \mathcal{G}_{1}}^{(p)}(F)(y)
$$

for nonzero real $q$ if the transforms exist.

For convenience, in this section we decided to work with $p=1$; however, in view of Remark 5.5, all of our results in this section also hold for $1<p \leq 2$.

Using the techniques similar to those used in the proof of Theorem 5.2, we obtain the following theorem.

Theorem 7.1. Let $k_{1}$ and $k_{2}$ be nonzero elements of $C_{a, b}^{*}[0, T]$, let $q_{0}$ be a positive real number, and let $F \in \mathcal{F}_{k_{1}, k_{2}}^{q_{0}}$ be given by equation (5.1). Then, for all real $q$ with $|q|>q_{0}$, the $L_{1}$ analytic Multi- $\left(\mathcal{Z}_{k_{1}}, \mathcal{Z}_{k_{2}}\right)$-GFFT, $\mathcal{M}_{q,\left(\mathcal{Z}_{k_{1}}, \mathcal{Z}_{k_{2}}\right)}^{(1)}(F)$ of F exists and is given by the formula

$$
\mathcal{M}_{q,\left(\mathcal{Z}_{k_{1}}, \mathcal{Z}_{k_{2}}\right)}^{(1)}(F)(y)=\int_{C_{a, b}^{\prime}[0, T]} \exp \left\{i(w, y)^{\sim}\right\} \Psi_{2}\left(-i q,-i q, k_{1}, k_{2} ; w\right) d f(w)
$$

for s-a.e. $y \in C_{a, b}[0, T]$, where $\Psi_{2}$ is given by equation (6.2).

For $k_{1}, k_{2} \in C_{a, b}^{\prime}[0, T]$ with $\left\|k_{j}\right\|_{C_{a, b}^{\prime}}>0$ and $j \in\{1,2\}$, let $\mathcal{Z}_{k_{1}}$ and $\mathcal{Z}_{k_{2}}$ be the Gaussian processes given by (3.1) respectively. Then the process

$$
\mathfrak{X}_{k_{1}, k_{2}}: C_{a, b}[0, T] \times C_{a, b}[0, T] \times[0, T] \rightarrow \mathbb{R}
$$

given by

$$
\mathfrak{X}_{k_{1}, k_{2}}\left(x_{1}, x_{2}, t\right):=\mathcal{Z}_{k_{1}}\left(x_{1}, t\right)+\mathcal{Z}_{k_{2}}\left(x_{2}, t\right)
$$

is also a Gaussian process with mean

$$
\mathfrak{m}_{k_{1}, k_{2}}(t):=\int_{C_{a, b}^{2}[0, T]} \mathfrak{X}_{k_{1}, k_{2}}\left(x_{1}, x_{2}, t\right) d(\mu \times \mu)\left(x_{1}, x_{2}\right)=\gamma_{k_{1}}(t)+\gamma_{k_{2}}(t)
$$

and variance

$$
\begin{aligned}
\mathfrak{v}_{k_{1}, k_{2}}(t): & =\int_{C_{a, b}^{2}[0, T]}\left(\mathfrak{X}_{k_{1}, k_{2}}\left(x_{1}, x_{2}, t\right)-\mathfrak{m}_{k_{1}, k_{2}}(t)\right)^{2} d(\mu \times \mu)\left(x_{1}, x_{2}\right) \\
& =\beta_{k_{1}}(t)+\beta_{k_{2}}(t)
\end{aligned}
$$

where $\gamma_{k}$ and $\beta_{k}$ are given by (3.2) and (3.3) respectively. In fact, the covariance of $\mathfrak{X}_{k_{1}, k_{2}}\left(x_{1}, x_{2}, \cdot\right)$ is given by

$$
\begin{aligned}
& \int_{C_{a, b}^{2}[0, T]}\left(\mathfrak{X}_{k_{1}, k_{2}}\left(x_{1}, x_{2}, s\right)-\mathfrak{m}_{k_{1}, k_{2}}(s)\right)\left(\mathfrak{X}_{k_{1}, k_{2}}\left(x_{1}, x_{2}, t\right)-\mathfrak{m}_{k_{1}, k_{2}}(t)\right) d(\mu \times \mu)\left(x_{1}, x_{2}\right) \\
& =\beta_{k_{1}}(\min \{s, t\})+\beta_{k_{2}}(\min \{s, t\}) \\
& =\mathfrak{v}_{k_{1}, k_{2}}(\min \{s, t\}) .
\end{aligned}
$$


Let $k_{1}$ and $k_{2}$ be elements of $C_{a, b}^{\prime}[0, T]$ (resp. $\left.C_{a, b}^{*}[0, T]\right)$ with $D k_{j}=h_{j}, j \in$ $\{1,2\}$. Then there exists a function $\mathbf{s}$ in $C_{a, b}^{\prime}[0, T]$ (resp. $\left.C_{a, b}^{*}[0, T]\right)$ such that

$$
(D \mathbf{s}(t))^{2}=h_{1}^{2}(t)+h_{2}^{2}(t)=\left(D k_{1}(t)\right)^{2}+\left(D k_{2}(t)\right)^{2}
$$

for $m_{|a|, b}$-a.e. $t \in[0, T]$. Note that the function 's' satisfying (7.2) is not unique. We will use the symbol $\mathbf{s}\left(k_{1}, k_{2}\right)$ for the functions ' $\mathbf{s}$ ' that satisfy (7.2) above.

Next we will consider a stochastic process associated with $\mathcal{Z}_{\mathbf{s}\left(k_{1}, k_{2}\right)}$. Define a process

$$
\mathcal{R}_{k_{1}, k_{2}}: C_{a, b}[0, T] \times[0, T] \rightarrow \mathbb{R}
$$

by

$$
\mathcal{R}_{k_{1}, k_{2}}(x, t):=\mathcal{Z}_{\mathbf{s}\left(k_{1}, k_{2}\right)}(x, t)+\mathcal{A}\left(k_{1}, k_{2}\right)(t),
$$

where

$$
\mathcal{A}\left(k_{1}, k_{2}\right)(t):=\int_{0}^{t}\left(D k_{1}(u)+D k_{2}(u)-D \mathbf{s}\left(k_{1}, k_{2}\right)(u)\right) d a(u) .
$$

Then $\mathcal{R}_{k_{1}, k_{2}}$ is a Gaussian process with mean

$$
\begin{aligned}
& \int_{C_{a, b}[0, T]} \mathcal{R}_{k_{1}, k_{2}}(x, t) d \mu(x) \\
& =\int_{C_{a, b}[0, T]} \mathcal{Z}_{\mathbf{s}\left(k_{1}, k_{2}\right)}(x, t) d \mu(x)+\mathcal{A}\left(k_{1}, k_{2}\right)(t) \\
& =\gamma_{k_{1}}(t)+\gamma_{k_{2}}(t) \\
& =\mathfrak{m}_{k_{1}, k_{2}}(t)
\end{aligned}
$$

and covariance

$$
\begin{aligned}
& \int_{C_{a, b}[0, T]}\left(\mathcal{R}_{k_{1}, k_{2}}(x, s)-\mathfrak{m}_{k_{1}, k_{2}}(s)\right)\left(\mathcal{R}_{k_{1}, k_{2}}(x, t)-\mathfrak{m}_{k_{1}, k_{2}}(t)\right) d \mu(x) \\
& =\int_{C_{a, b}[0, T]}\left(\mathcal{Z}_{\mathbf{s}\left(k_{1}, k_{2}\right)}(x, s)-\gamma_{\mathbf{s}\left(k_{1}, k_{2}\right)}(s)\right)\left(\mathcal{Z}_{\mathbf{s}\left(k_{1}, k_{2}\right)}(x, t)-\gamma_{\mathbf{s}\left(k_{1}, k_{2}\right)}(t)\right) d \mu(x) \\
& =\beta_{\mathbf{s}\left(k_{1}, k_{2}\right)}(\min \{s, t\}) \\
& =\int_{0}^{\min \{s, t\}}\left[D \mathbf{s}\left(k_{1}, k_{2}\right)(u)\right]^{2} d b(u) \\
& =\int_{0}^{\min \{s, t\}}\left(\left[D k_{1}(u)\right]^{2}+\left[D k_{2}(u)\right]^{2}\right) d b(u) \\
& =\beta_{k_{1}}(\min \{s, t\})+\beta_{k_{2}}(\min \{s, t\}) \\
& =\mathfrak{v}_{k_{1}, k_{2}}(\min \{s, t\}) .
\end{aligned}
$$

Also, $\mathcal{R}_{k_{1}, k_{2}}(\cdot, t)$ is stochastically continuous in $t$ on $[0, T]$. 
From these facts, one can see that the Gaussian processes $\mathfrak{X}_{k_{1}, k_{2}}$ and $\mathcal{R}_{k_{1}, k_{2}}$ have the same distribution and that for any random variable $F$ on $C_{a, b}[0, T]$,

$$
\begin{aligned}
& \int_{C_{a, b}^{2}[0, T]} F\left(\mathfrak{X}_{k_{1}, k_{2}}\left(x_{1}, x_{2}, \cdot\right)\right) d \mu^{2}\left(x_{1}, x_{2}\right) \\
& \equiv \int_{C_{a, b}[0, T]}\left[\int_{C_{a, b}[0, T]} F\left(\mathfrak{X}_{k_{1}, k_{2}}\left(x_{1}, x_{2}, \cdot\right)\right) d \mu\left(x_{1}\right)\right] d \mu\left(x_{2}\right) \\
& \stackrel{*}{=} \int_{C_{a, b}[0, T]} F\left(\mathcal{R}_{k_{1}, k_{2}}(x, \cdot)\right) d \mu(x),
\end{aligned}
$$

where by $\stackrel{*}{=}$ we mean that if either side exists, both sides exist and equality holds. Remark 7.2. In the case of $a(t) \equiv 0$, we observe that $\mathcal{A}\left(k_{1}, k_{2}\right)(t) \equiv 0$ and so $\mathcal{R}_{k_{1}, k_{2}}(x, t)=\mathcal{Z}_{\mathbf{s}\left(k_{1}, k_{2}\right)}(x, t)$. Thus equation (7.5) can be rewritten as

$$
\int_{C_{a, b}^{2}[0, T]} F\left(\mathfrak{X}_{k_{1}, k_{2}}\left(x_{1}, x_{2}, \cdot\right)\right) d \mu^{2}\left(x_{1}, x_{2}\right) \stackrel{*}{=} \int_{C_{a, b}[0, T]} F\left(\mathcal{Z}_{\mathbf{s}\left(k_{1}, k_{2}\right)}(x, t)\right) d \mu(x) .
$$

Remark 7.3. In [13], the authors investigated a rotation property of the function space measure $\mu$. The result is summarized as follows: for a measurable functional $F$, and all nonzero real $p$ and $q$,

$$
\begin{aligned}
& \int_{C_{a, b}^{2}[0, T]} F\left(p x_{1}+q x_{2}\right) d \mu^{2}\left(x_{1}, x_{2}\right) \\
& \stackrel{*}{=} \int_{C_{a, b}[0, T]} F\left(\sqrt{p^{2}+q^{2}} x+\left(p+q-\sqrt{p^{2}+q^{2}}\right) a\right) d \mu(x) .
\end{aligned}
$$

Let $k_{1}(t)=p b(t)$ and $k_{2}(t)=q b(t)$ on $[0, T]$. Then from equations (3.1), (7.3) and (7.5), we can obtain equation (7.6). But, by the observation of equation (7.2) above, we obtain the alternative result

$$
\begin{aligned}
& \int_{C_{a, b}^{2}[0, T]} F\left(p x_{1}+q x_{2}\right) d \mu^{2}\left(x_{1}, x_{2}\right) \\
& \stackrel{*}{=} \int_{C_{a, b}[0, T]} F\left(-\sqrt{p^{2}+q^{2}} x+\left(p+q+\sqrt{p^{2}+q^{2}}\right) a\right) d \mu(x) .
\end{aligned}
$$

Remark 7.4. Note that if $k_{1}, k_{2} \in C_{a, b}^{*}[0, T]$, then $\mathcal{R}_{k_{1}, k_{2}}$ is a continuous process.

Theorem 7.5. Let $k_{1}, k_{2}, q_{0}$ and $F \in \mathcal{F}_{k_{1}, k_{2}}^{q_{0}}$ be as in Theorem \%.1. Let $\mathbf{s}\left(k_{1}, k_{2}\right)$ be an element of $C_{a, b}^{*}[0, T]$ which satisfies equation (7.2). Then, for all real $q$ with $|q|>q_{0}$, the $L_{1}$ analytic $\mathcal{R}_{k_{1}, k_{2}}-G F F T, T_{q, \mathcal{R}_{k_{1}, k_{2}}}^{(1)}(F)$ of $F$ exists and is given by the right hand side of equation (7.1), i.e.,

$$
T_{q, \mathcal{R}_{k_{1}, k_{2}}^{(1)}}^{(F)}(y)=\mathcal{M}_{q,\left(\mathcal{Z}_{k_{1}}, \mathcal{Z}_{k_{2}}\right)}^{(1)}(F)(y)
$$

for s-a.e. $y \in C_{a, b}[0, T]$. 
Proof. In view of the definition of the $L_{1}$ analytic $\mathcal{G}$-GFFT and Theorem 7.1 , it suffices to show that for all $\lambda>0$,

$$
T_{\lambda, \mathcal{R}_{k_{1}, k_{2}}}(F)(y)=\mathcal{M}_{\lambda,\left(\mathcal{Z}_{k_{1}}, \mathcal{Z}_{k_{2}}\right)}(F)(y)
$$

for s-a.e. $y \in C_{a, b}[0, T]$.

First, using (7.3), (5.1), the Fubini theorem, and (3.7) with $\mathcal{Z}_{k}$ replaced with $\mathcal{Z}_{\mathbf{s}\left(k_{1}, k_{2}\right)}$, it follows that for all $\lambda>0$ and s-a.e. $y \in C_{a, b}[0, T]$,

$$
\begin{aligned}
& T_{\lambda, \mathcal{R}_{k_{1}, k_{2}}}(F)(y) \\
& =I_{\mathcal{R}_{k_{1}, k_{2}, x}}\left[F\left(y+\lambda^{-1 / 2} \mathcal{R}_{k_{1}, k_{2}}(x, \cdot)\right)\right] \\
& =\int_{C_{a, b}^{\prime}[0, T]} \exp \left\{i(w, y)^{\sim}+i \lambda^{-1 / 2}\left(w, \mathcal{A}\left(k_{1}, k_{2}\right)\right)^{\sim}\right\} \\
& \quad \times I_{\mathcal{Z}_{\mathbf{s}\left(k_{1}, k_{2}\right)}, x}\left[\exp \left\{i \lambda^{-1 / 2}\left(w, \mathcal{Z}_{\mathbf{s}\left(k_{1}, k_{2}\right)}(x, \cdot)\right)^{\sim}\right\}\right] d f(w) \\
& =\int_{C_{a, b}^{\prime}[0, T]} \exp \left\{i(w, y)^{\sim}+i \lambda^{-1 / 2}\left(w, \mathcal{A}\left(k_{1}, k_{2}\right)\right)^{\sim}\right. \\
& \left.\quad-\frac{1}{2 \lambda}\left\|w \odot \mathbf{s}\left(k_{1}, k_{2}\right)\right\|_{C_{a, b}^{\prime}}^{2}+i \lambda^{-1 / 2}\left(w \odot \mathbf{s}\left(k_{1}, k_{2}\right), a\right)_{C_{a, b}^{\prime}}\right\} d f(w) .
\end{aligned}
$$

Next, using (7.2), (7.4), and (3.4), we observe that

$$
\begin{aligned}
\| w \odot \mathbf{s} & \left(k_{1}, k_{2}\right) \|_{C_{a, b}^{\prime}}^{2}=\left(w \odot \mathbf{s}\left(k_{1}, k_{2}\right), w \odot \mathbf{s}\left(k_{1}, k_{2}\right)\right)_{C_{a, b}^{\prime}} \\
& =\int_{0}^{T}(D w(t))^{2}\left(D \mathbf{s}\left(k_{1}, k_{2}\right)(t)\right)^{2} d b(t) \\
& =\int_{0}^{T}(D w(t))^{2}\left(D k_{1}(t)\right)^{2} d b(t)+\int_{0}^{T}(D w(t))^{2}\left(D k_{2}(t)\right)^{2} d b(t) \\
& =\left\|w \odot k_{1}\right\|_{C_{a, b}^{\prime}}^{2}+\left\|w \odot k_{2}\right\|_{C_{a, b}^{\prime}}^{2}
\end{aligned}
$$

and

$$
\begin{aligned}
& \left(w \odot \mathbf{s}\left(k_{1}, k_{2}\right), a\right)_{C_{a, b}^{\prime}}+\left(w, \mathcal{A}\left(k_{1}, k_{2}\right)\right)^{\sim} \\
& =\left(w \odot \mathbf{s}\left(k_{1}, k_{2}\right), a\right)_{C_{a, b}^{\prime}} \\
& +\int_{0}^{T} D w(t) d\left[\int_{0}^{t}\left(D k_{1}(u)+D k_{2}(u)-D \mathbf{s}\left(k_{1}, k_{2}\right)(u)\right) d a(u)\right] \\
& =\left(w \odot \mathbf{s}\left(k_{1}, k_{2}\right), a\right)_{C_{a, b}^{\prime}}-\int_{0}^{T} D w(t) D \mathbf{s}\left(k_{1}, k_{2}\right)(t) d a(t) \\
& +\int_{0}^{T} D w(t) D k_{1}(t) d a(t)+\int_{0}^{T} D w(t) D k_{2}(t) d a(t) \\
& =\int_{0}^{T} D\left(w \odot k_{1}\right)(t) D a(t) d b(t)+\int_{0}^{T} D\left(w \odot k_{2}\right)(t) D a(t) d b(t) \\
& =\left(w \odot k_{1}, a\right)_{C_{a, b}^{\prime}}+\left(w \odot k_{2}, a\right)_{C_{a, b}^{\prime}} \text {. }
\end{aligned}
$$


Finally, using equations (7.7), (7.8), (7.9), (6.2), and (7.1), it follows that

$$
\begin{aligned}
T_{\lambda, \mathcal{R}_{k_{1}, k_{2}}}(F)(y)= & \int_{C_{a, b}^{\prime}[0, T]} \exp \left\{i(w, y)^{\sim}-\frac{1}{2 \lambda} \sum_{j=1}^{2}\left\|w \odot k_{j}\right\|_{C_{a, b}^{\prime}}^{2}\right. \\
& \left.+i \lambda^{-1 / 2} \sum_{j=1}^{2}\left(w \odot k_{j}, a\right)_{C_{a, b}^{\prime}}\right\} d f(w) \\
= & \int_{C_{a, b}^{\prime}[0, T]} \exp \left\{i(w, y)^{\sim}\right\} \Psi_{2}\left(\lambda, \lambda, k_{1}, k_{2} ; w\right) d f(w) \\
= & \mathcal{M}_{\lambda,\left(\mathcal{Z}_{k_{1}}, \mathcal{Z}_{k_{2}}\right)}(F)(y)
\end{aligned}
$$

for s-a.e. $y \in C_{a, b}[0, T]$.

The following theorem follows from the previous results, and the use of mathematical induction.

Theorem 7.6. Given $k_{j} \in C_{a, b}^{*}[0, T] \backslash\{0\}, j \in\{1, \ldots, n\}$, let $\mathcal{Z}_{k_{j}}$ be the Gaussian processes given by (3.1), and let $\mathcal{R}_{k_{1}, \ldots, k_{n}}: C_{a, b}[0, T] \times[0, T] \rightarrow \mathbb{R}$ be the Gaussian process given by

$$
\mathcal{R}_{k_{1}, \ldots, k_{n}}(x, t):=\mathcal{Z}_{\mathbf{s}\left(k_{1}, \ldots, k_{n}\right)}(x, t)+\int_{0}^{t}\left[\sum_{j=1}^{n} D k_{j}(u)-D \mathbf{s}\left(k_{1}, \ldots, k_{n}\right)(u)\right] d a(u),
$$

where $\mathbf{s}\left(k_{1}, \ldots, k_{n}\right)$ is an element of $C_{a, b}^{*}[0, T]$ which satisfies the condition

$$
\left[D \mathbf{s}\left(k_{1}, \ldots, k_{n}\right)\right]^{2}=\sum_{j=1}^{n}\left[D k_{j}\right]^{2}
$$

for $m_{|a|, b}$-a.e. on $[0, T]$. Given $q_{0}>0$, let $F \in \mathcal{F}_{k_{1}, \ldots, k_{n}}^{q_{0}}$ be given by equation (5.1). Then, for all real $q$ with $|q|>q_{0}$, the $L_{1}$ analytic $\mathcal{R}_{k_{1}, \ldots, k_{n}}-G F F T, T_{q, \mathcal{R}_{k_{1}, \ldots, k_{n}}}^{(1)}(F)$, and the $L_{1}$ analytic Multi- $\left(\mathcal{Z}_{k_{1}}, \ldots, \mathcal{Z}_{k_{n}}\right)$-GFFT, $\mathcal{M}_{q,\left(\mathcal{Z}_{k_{1}}, \ldots, \mathcal{Z}_{k_{n}}\right)}^{(1)}(F)$ of $F$ exist and

$$
\mathcal{M}_{q,\left(\mathcal{Z}_{k_{1}}, \ldots, \mathcal{Z}_{k_{n}}\right)}^{(1)}(F)(y)=T_{q, \mathcal{R}_{k_{1}, \ldots, k_{n}}^{(1)}}^{(F)}(y)
$$

for s-a.e. $y \in C_{a, b}[0, T]$. Also, both expressions in (7.10) are given by the right hand side of equation (6.4) with $q=q_{1}=\cdots=q_{n}$.

Remark 7.7. When $a(t) \equiv 0$ and $b(t)=t$ on $[0, T]$, the function space $C_{a, b}[0, T]$ reduces to the classical Wiener space $C_{0}[0, T]$. In this case, equation $(7.10)$ can be rewritten as

$$
\mathcal{M}_{q,\left(\mathcal{Z}_{k_{1}}, \ldots, \mathcal{Z}_{k_{n}}\right)}^{(1)}(F)(y)=T_{q, \mathcal{Z}_{\mathbf{s}\left(k_{1}, \ldots, k_{n}\right)}^{(1)}}^{(F)}(y)
$$

for all functionals $F \in \mathcal{F}\left(C_{0}[0, T]\right)$ and s-a.e. $y \in C_{0}[0, T]$ in view of Remarks 5.1 and 7.2. Thus most of the results in [10] follow immediately from the results in this paper.

Acknowledgement. The authors would like to express their gratitude to the editor and the referees for their valuable comments and suggestions which have improved the original paper. 


\section{REFERENCES}

1. M.D. Brue, A functional transform for Feynman integrals similar to the Fourier transform, Thesis, University of Minnesota, Minneapolis, 1972.

2. R.H. Cameron, The ILSTOW and Feynman integrals, J. Analyse Math. 10 (1962-63), 287361.

3. R.H. Cameron and D.A. Storvick, An $L_{2}$ analytic Fourier-Feynman transform, Michigan Math. J. 23 (1976), 1-30.

4. R.H. Cameron and D.A. Storvick, Some Banach algebras of analytic Feynman integrable functionals, Analytic Functions, 18-67, Lecture Notes in Math. vol. 798, Springer, Berlin, 1980.

5. K.S. Chang, D.H. Cho, B.S. Kim, T.S. Song and I. Yoo, Relationships involving generalized Fourier-Feynman transform, convolution and first variation, Integral Transform Spec. Funct. 16 (2005), 391-405.

6. S.J. Chang, J.G. Choi and D. Skoug, Integration by parts formulas involving generalized Fourier-Feynman transforms on function space, Trans. Amer. Math. Soc. 355 (2003), 29252948.

7. S.J. Chang, J.G. Choi and D. Skoug, Evaluation formulas for conditional function space integrals I, Stoch. Anal. Appl. 25 (2007), 141-168.

8. S.J. Chang and D. Skoug, Generalized Fourier-Feynman transforms and a first variation on function space, Integral Transforms Spec. Funct. 14 (2003), 375-393.

9. J.G. Choi, H.S. Chung and S.J. Chang, Sequential generalized transforms on function space, Abstract and Applied Analysis 2013 (2013), Article ID 565832, 12 pages.

10. J.G. Choi, D. Skoug and S.J. Chang, A multiple generalized Fourier-Feynman transform via a rotation on Wiener space, Int. J. Math. 23 (2012), Article ID: 1250068, 20 pages.

11. D.M. Chung and J.H. Lee, Generalized Brownian motions with application to finance, J. Korean Math. Soc. 43 (2006), 357-371.

12. D.M. Chung and C. Park and D. Skoug, Generalized Feynman integrals via conditional Feynman integrals, Michigan Math. J. 40 (1993), 377-391.

13. H.S. Chung, J.G. Choi and S.J. Chang, A Fubini theorem on a function space and its applications, Banach J. Math. Anal. 7 (2013), 173-185.

14. T.S. Chung and U.C. Ji, Gaussian white noise and applications to finance, Quantum Information and Complexity (T. Hida, K. Saitô, S. Si, Eds.), 179-201, World Scientific, Singapore, 2004.

15. D. Cohn, Measure Theory (Second edition), Birkhäuser, Boston, 2013.

16. M.K. Im and J.-H. Kim, Stochastic calculus for analogue of Wiener process, J. Korea Soc. Math. Educ. Ser. B: Pure Appl. Math. 14 (2007), 335-354.

17. G.W. Johnson, The equivalence of two approaches to the Feynman integral, J. Math. Phys. 23 (1982), 2090-2096.

18. G.W. Johnson and M.L. Lapidus, The Feynman Integral and Feynman's Operational Calculus, Clarendon Press, Oxford, 2000.

19. G.W. Johnson and D.L. Skoug, An $L_{p}$ analytic Fourier-Feynman transform, Michigan Math. J. 26 (1979), 103-127.

20. G. Kallianpur and C. Bromley, Generalized Feynman integrals using analytic continuation in several complex variables, Stochastic Analysis and Applications, 217-267, Marcel Dekker, Inc., New York, 1984.

21. J.H. Lee, The linear space of generalized Brownian motions with application, Proc. Amer. Math. Soc. 133 (2005), 2147-2155.

22. C. Park and D. Skoug, Generalized Feynman integrals: The $\mathcal{L}\left(L_{2}, L_{2}\right)$ theory, Rocky Mountain J. Math. 25 (1995), 739-756.

23. C. Park and D. Skoug, Conditional Fourier-Feynman transforms and conditional convolution products, J. Korean Math. Soc. 38 (2001), 61-76.

24. W. Rudin, Real and Complex Analysis (Third edition), McGraw-Hill, New York, 1987. 
25. D. Skoug and D. Storvick, A survey of results involving transforms and convolutions in function space, Rocky Mountain J. Math. 34 (2004), 1147-1175.

26. J. Yeh, Singularity of Gaussian measures on function spaces induced by Brownian motion processes with non-stationary increments, Illinois J. Math. 15 (1971), 37-46.

27. J. Yeh, Stochastic Processes and the Wiener Integral, Marcel Dekker, Inc., New York, 1973.

Department of Mathematics, Dankook University, Cheonan 330-714, Korea.

E-mail address: sejchang@dankook.ac.kr

E-mail address: jgchoi@dankook.ac.kr

E-mail address: ayko@dankook.ac.kr 